\title{
Mind the GAPs: insights into intestinal epithelial barrier maintenance and luminal antigen delivery
}

\author{
MJ Miller ${ }^{1,2}$, KA Knoop ${ }^{1}$ and RD Newberry ${ }^{1}$
}

The small intestine (SI) epithelium is a major interface between the body and the environment. In addition to nutrient absorption, this single layer epithelium must act as a barrier to pathogenic infection while allowing the underlying immune system to selectively sample antigens from the normal flora and the diet to promote homeostasis. How the epithelium simultaneously performs these opposing tasks is a fundamental question in mucosal immunology. Recently, it was discovered that when goblet cells (GCs) secrete, they form goblet cell-associated antigen passages (GAPs) that can deliver small soluble luminal antigens to lamina propria (LP) dendritic cells (DCs). ${ }^{1}$ This striking observation suggests that GCs have a previously unappreciated role in regulating adaptive immune responses at the mucosa and provides important insight into how the epithelium can maintain its barrier function, while allowing LP-DCs to sample the intestinal contents.

The SI LP is densely populated by macrophages and DCs, with various subsets contributing to inflammation, tolerance and adaptive immunity. Antigen presentation is a central function of DCs, thus it has been assumed that
LP-DCs have the ability to capture antigen from the epithelium and even the lumen of the intestine. Several mechanisms have been identified for the delivery of luminal antigens to LP-DCs, including paracellular leak, villous microfold cells ( $\mathrm{M}$ cells), and trans-epithelial dendrite (TED) extension by LP-DCs. However, the relative contribution of each of these pathways and the context in which they contribute to downstream immune responses has been largely unexplored. Using in vivo two-photon microscopy, McDole et al. examined the steadystate uptake of fluorescently labeled soluble antigens across the SI epithelium and found that a subset of epithelial cells rapidly filled with luminal antigen. Time-lapse movies showed that LP-DCs actively probed these epithelial cells and could acquire antigen from them directly, whereas antigen capture via paracellular leak was undetectable microscopically. Subsequently, the antigen-containing epithelial cells were shown to be GCs, and multiple approaches confirmed that GCs effectively delivered small $(<70 \mathrm{kD})$ soluble luminal antigens to underlying LP-DCs. In mice that lack GCs, luminal antigen acquisition and presentation by LP-DCs was not detectable, implying that this mechanism is an important, and perhaps dominant, pathway for steady-state luminal antigen delivery to LP-DCs. This antigen delivery pathway was termed goblet cell-associated antigen passages, or GAPs, ${ }^{1}$ and these observations suggested a provocative new function for GCs at the frontline of intestinal immunology. However, many important questions remain, such as the molecular mechanisms and regulation of GCmediated antigen delivery, and the role of GAPs in intestinal immunity and tolerance.

Insight into how GCs form GAPs came from the observation that GAPs rapidly increased in response to the cholinergic agonist carbamylcholine, ${ }^{1}$ a well-known GC secretagogue, thus suggesting that GAP formation was linked with GC secretion. Studies of intestinal GC mucus secretion performed three decades ago provide the basis to begin to understand how GAPs form and are regulated. $^{2,3}$ Intestinal GCs secrete via two pathways, primary exocytosis (PE), which is regulated by cAMP and induces the secretion of single vesicles of mucin and compound exocytosis (CE), which is regulated by intracellular $\mathrm{Ca}^{2+}$ and induces the fusion and secretion of multiple preformed apically stored mucin granules. ${ }^{2,4}$ Studies of pancreatic acinar cells revealed that during CE the fused vesicles transiently remain in a stable open form and exposed to the extracellular environment for minutes allowing extracellular dyes into the cell, ${ }^{5}$ which could be related to what was observed when intestinal GCs get filled with antigen to form GAPs. Consistent with $\mathrm{CE}$, but not PE, being linked to GAP formation, a dramatic increase in GAPs

${ }^{1}$ Department of Internal Medicine, Washington University School of Medicine, St Louis, MO, USA and ${ }^{2}$ Department of Pathology and Immunology, Washington University School of Medicine, St Louis, MO, USA. Correspondence: RD Newberry (rnewberry@wustl.edu) 

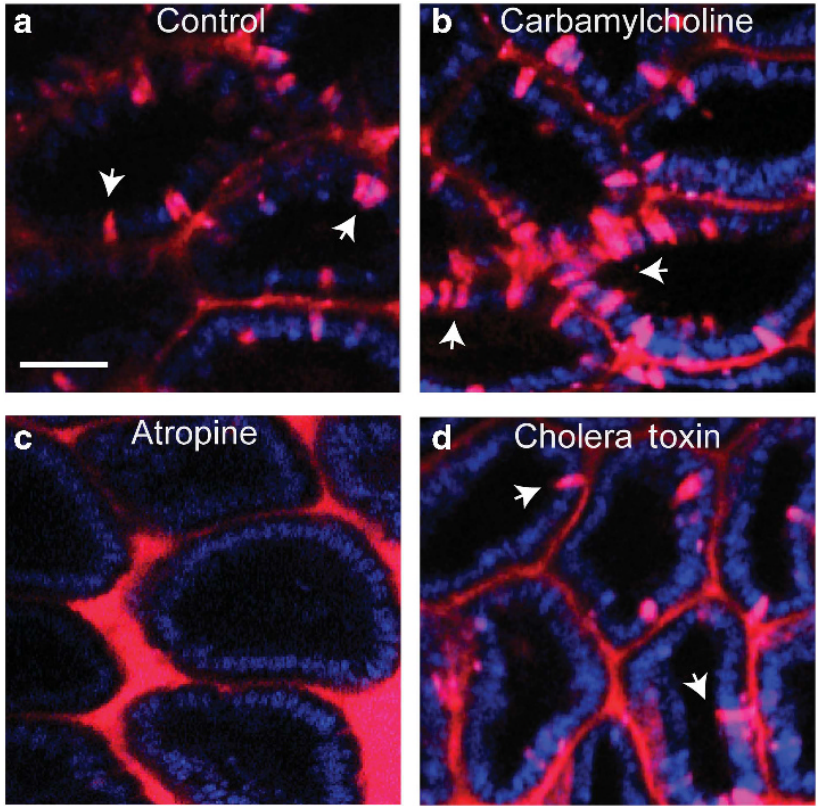

Figure 1 Induction and inhibition of GAPs. Two-photon microscopy of the small intestine of anesthetized mice given intraluminal $10 \mathrm{kD}$ fluorescent dextran (red) and DAPI (nuclear dye; blue) revealed (a) the presence of columns of dextran crossing the epithelium, or GAPs (white arrows) in the steady state. (b) GAPs were increased in response to the cholinergic agonist carbamylcholine, $3 \mu \mathrm{g}$ injected subcutaneously during imaging and (c) inhibited by the pan-muscarinic acetylcholine receptor agonist atropine, $12 \mathrm{mg}$ injected intraperitoneally $2 \mathrm{~h}$ before imaging. (d) GAP formation was unaffected by cholera toxin, $5 \mu \mathrm{g}$ intraluminally before imaging, which induces $\mathrm{GC}$ secretion via $\mathrm{PE}$. DAPI, 4',6-diamidino-2-phenylindole; GAP, goblet cell-associated antigen passage; GC, goblet cell; $\mathrm{PE}$, primary exocytosis. Bar $=25 \mu \mathrm{m}$.

is seen after stimulation with the cholinergic agonist, ${ }^{1}$ carbamylcholine, which induces $\mathrm{Ca}^{2+}$-mediated $\mathrm{CE},{ }^{2,3}$ but not by cholera toxin, which induces cAMP-mediated PE (Figure 1). Multiple stimuli inducing GC secretion via CE have been identified, ${ }^{3}$ but whether these will also induce GAP formation and antigen delivery across the epithelium remains to be investigated. Linking GAP formation with one secretory pathway, but not both, might allow antigen delivery to be regulated independent of mucus secretion. This would allow GCs to maintain the mucus barrier and not deliver luminal antigens to underlying LP-DCs, which would be beneficial when the luminal environment and contents are deemed unfavorable. Moreover, the ability of GCs to secrete mucus via $\mathrm{PE}$ and not form GAPs, may explain the absence of GAPs in the colon, where GCs are plentiful and mucus abundant.

In the basal state, GAP formation is driven by acetylcholine as demonstrated by the ability of atropine, a pan-muscarinic acetylcholine receptor (mAChR) antagonist to inhibit GAPs (Figure 1). GCs secrete mucus upon direct stimulation with cholinergic agonists in vitro, ${ }^{6}$ implying that acetylcholine could act directly upon the GCs via mAChRs to form GAPs, suggesting a working model on how GAPs may form (Figure 2). Interestingly not all SI GCs formed GAPs following stimulation by exogenous cholingeric agonists. This may be related to a refractory period following secretion, in which GCs can no longer respond to these stimuli and secrete by CE, or it could suggest a heterogeneity within GCs where only a subset of GCs have the capacity to form GAPs. The latter possibility is consistent with observations that GAPs preferentially formed on the upper portion of villi, which could suggest that more differentiated or mature GCs have the capacity to form GAPs. This might also be an explanation for the absence of GAPs in the colon, as these GCs may be fundamentally different to those that develop in the SI.

The role of GAPs in intestinal immunity is largely unknown, but several observations suggest that GAPs are a mechanism to "educate" the immune system about innocuous luminal antigens encountered during homeostasis. GAPs were present in disease-free human SI resection specimens and in the SI of all healthy mouse strains examined, indicating that GAPs are a component of normal physiology. In addition, GAPs preferentially delivered small, soluble dextrans and protein antigens, but did not efficiently transport large $(2,000 \mathrm{kD})$ dextrans or inert particles as small as $20 \mathrm{~nm} .{ }^{1}$ The characteristics of the substances GAPs delivered suggest that they are more likely to deliver small soluble antigens derived from food and microbiota, as opposed to whole live bacteria. However, inert particles are markedly different from live bacteria, and a recent study reported that Listeria monocytogenes exploits GC to cross the SI epithelial barrier, ${ }^{7}$ raising the intriguing notion that GAPs may be hijacked by pathogens to provide a portal of entry.

Further clues for the role of GAPs in intestinal immunity come from observations that GAPs delivered antigen preferentially to CD103+ LP-DCs. ${ }^{1}$ The evidence for selective delivery is based on the direct in vivo observation of antigen capture by LP-DCs using two-photon microscopy and the ex vivo analysis of the antigen capture and presentation capacity by LP-DC subsets isolated from mice that were given luminal antigen. Although the functional roles of different LP-DC subsets is still an area of active investigation, multiple studies have demonstrated that CD103 + LP-DCs can induce characteristic homeostatic immune responses, including imprinting gut-homing molecules on responding lymphocytes, facilitating the conversion of Foxp3 Tregs, and promoting IgA production. ${ }^{8}$ Given that antigen delivery to LP-DCs was undetectable in the absence of GAPs, and that the nonPeyer's patch epithelium has been implicated as the site of antigen delivery to DCs that migrate to the mesenteric lymph nodes (MLN) and promote oral tolerance, ${ }^{9,10}$ GAPs probably have a role in homeostatic responses. In further support of this, GAPs were found to transfer GC proteins and luminal 


\section{Goblet cell-associated antigen passage}

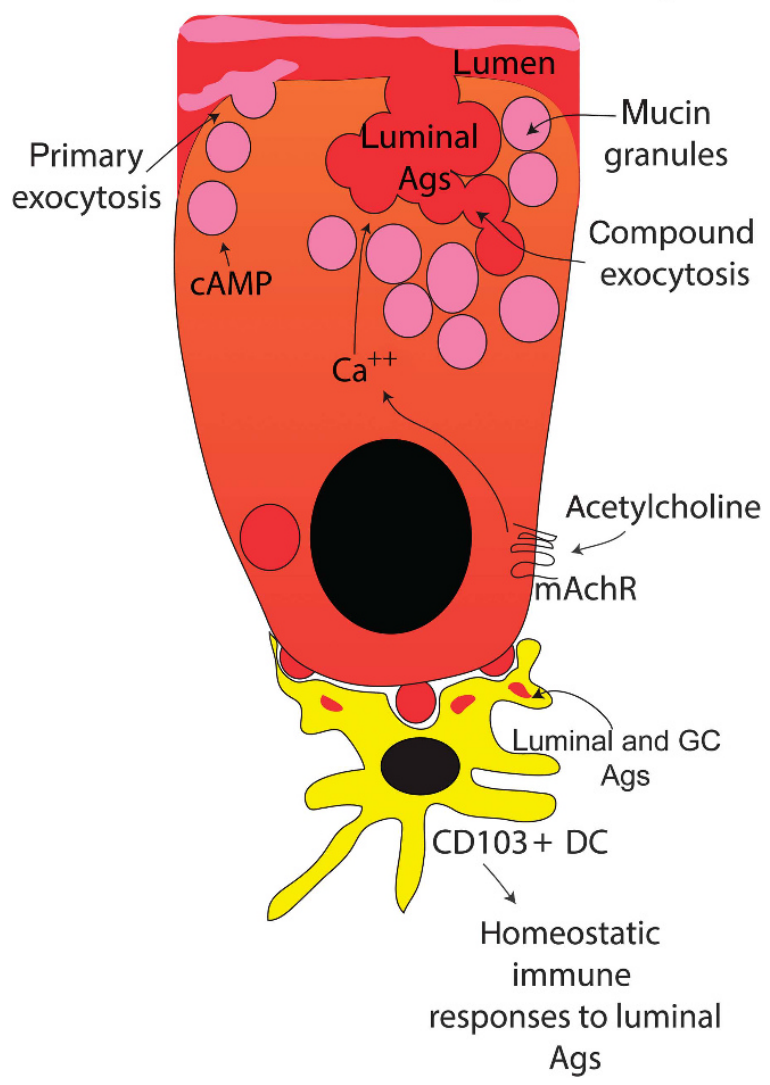

Figure 2 Working model of GAP formation. Muscarinic acetylcholine receptors (mAChR) on GCs sense acetylcholine and undergo calcium-mediated compound exocytosis. During compound exocytosis, holes are formed in the apical membrane and luminal antigen fills the cell. Antigen and GC proteins are acquired by CD103 + LP-DCs nearby or in direct contact with the GC. Secretion via cAMP-driven primary exocytosis does not induce GAP formation, and may allow GCs to secrete mucus to maintain barrier integrity in situations where antigen delivery is not wanted. DC, dendritic cell; GAP, goblet cell-associated antigen passage; GC, goblet cell; LP, lamina propria.

antigens to LP-DCs, ${ }^{1}$ and recent studies identified that the GC protein mucin 2 imprinted CD103 + LP-DCs with immunoregulatory properties. ${ }^{11}$ Together these observations indicate that GAPs can act as a source for self and luminal antigens, as well as deliver immunoregulatory stimuli to LP-DCs in the steady-state. Moreover, GC deficiency and dysfunction is linked with the development of intestinal inflammation, ${ }^{12-14}$ consistent with a role for GAPs in preserving homeostasis. However, to what extent the loss of GAPs alone contributes to inflammation is unclear, as these conditions will also have defective mucus secretion and alterations in mucosal barrier function.

While many questions remain unanswered, it is clear that the ability of GCs to form GAPs adds an entirely new dimension to the GC beyond mucus barrier maintenance. Because not all secretagogues and forms of secretion induce GAPs, antigen delivery could be regulated without compromising maintenance of the mucus barrier. Furthermore, the idea that small soluble luminal antigens and GC proteins, such as the immunoregulatory protein mucin 2 , are delivered simultaneously to LP-DCs suggests that GAPs may be uniquely suited to promote tolerance to innocuous antigens from the gut lumen. Therefore, the abundant, but often overlooked GC, may have a surprisingly complex and interesting role in mucosal immunology.

\section{ACKNOWLEDGMENTS}

Supported by grants: DK64798-RDN, Al009550RDN and MJM, DK097317-RDN and MJM, and DK097893 KAK. The two-photon imaging was performed at the In Vivo Imaging Core at Washington University School of Medicine, which is supported in part by the Washington University Digestive Diseases Research Center Core (DDRCC), funded by NIH grant P30 DK052574.

\section{DISCLOSURE}

The authors declared no conflict of interest.

c) 2014 Society for Mucosal Immunology

\section{REFERENCES}

1. McDole, J.R. et al. Goblet cells deliver luminal antigen to CD103 + dendritic cells in the small intestine. Nature 483, 345-349 (2012).

2. Specian, R.D. \& Neutra, M.R. Mechanism of rapid mucus secretion in goblet cells stimulated by acetylcholine. J. Cell Biol. 85, 626-640 (1980).

3. Neutra, M.R., O'Malley, L.J. \& Specian, R.D. Regulation of intestinal goblet cell secretion. II. A survey of potential secretagogues. Am. J. Physiol. 242, G380-G387 (1982).

4. Epple, H.J. et al. Differential stimulation of intestinal mucin secretion by cholera toxin and carbachol. Pflugers Arch. 433, 638-647 (1997).

5. Pickett, J.A. \& Edwardson, J.M. Compound exocytosis: mechanisms and functional significance. Traffic 7, 109-116 (2006).

6. Shatos, M.A., Kano, H., Rubin, P., Garza, G. \& Dartt, D.A. Isolation and characterization of human goblet cells in vitro: regulation of proliferation and activation of mitogen-activated protein kinase by EGF and carbachol. Adv. Exp. Med. Biol. 506, 301-305 (2002).

7. Nikitas, G. et al. Transcytosis of Listeria monocytogenes across the intestinal barrier upon specific targeting of goblet cell accessible E-cadherin. J. Exp. Med. 208, 2263-2277 (2011).

8. Scott, C.L., Aumeunier, A.M. \& Mowat, A.M. Intestinal CD103 + dendritic cells: master regulators of tolerance? Trends Immunol. 32, 412-419 (2011).

9. Spahn, T.W. \& Kucharzik, T. Modulating the intestinal immune system: the role of lymphotoxin and GALT organs. Gut 53, 456-465 (2004).

10. Worbs, T. et al. Oral tolerance originates in the intestinal immune system and relies on antigen carriage by dendritic cells. J. Exp. Med. 203, 519-527 (2006).

11. Shan, M. et al. Mucus enhances gut homeostasis and oral tolerance by delivering immunoregulatory signals. Science 342, 447-453 (2013).

12. Dvorak, A.M. \& Dickersin, G.R. Crohn's disease: transmission electron microscopic studies. I. Barrier function. Possible changes related to alterations of cell coat, mucous coat, epithelial cells, and Paneth cells. Hum. Pathol. 11, 561-571 (1980).

13. Tytgat, K.M., van der Wal, J.W., Einerhand, A.W., Buller, H.A. \& Dekker, J. Quantitative analysis of MUC2 synthesis in ulcerative colitis. Biochem. Biophys. Res. Commun. 224, 397-405 (1996).

14. Van der Sluis, M. et al. Muc2-deficient mice spontaneously develop colitis, indicating that MUC2 is critical for colonic protection. Gastroenterology 131, 117-129 (2006). 